\title{
Endovascular Recanalization of Life-Threatening Cerebral Venous Thrombosis Secondary to latrogenic Intracranial Hypotension
}

Hyesoo Kwon, MD; Yu Hun Jeong, MD; Moonkyung Choi, MD; Sohyeon Kim, MD; Hyungjong Park, MD; Keun Tae Kim, MD (D); Jeong-Ho Hong, MD, PhD (D); Sung-Il Sohn MD, PhD (iD)

Department of Neurology, Keimyung University School of Medicine, Daegu, Korea

Intracranial hypotension (IH) is characterized by postural headache, subdural effusion, cranial nerve palsy, and altered mental status. Cerebral venous thrombosis (CVT) is a rare complication secondary to IH. Anticoagulation is currently the mainstay of treatment of CVT. However, mechanical thrombectomy (MT) may be needed for patients who develop progressive neurologic worsening despite adequate anticoagulation. Here, we report a case of CVT secondary to iatrogenic IH that was successfully treated using MT.

J Neurosonol Neuroimag 2020;12(1):62-65

Key Words: Brain infarction; Venous thrombosis; Intracranial hypotension; Thrombectomy
Received: April 9, 2020

Revised: May 8, 2020

Accepted: May 26, 2020

Correspondence:

Hyungjong Park, MD

Department of Neurology, Keimyung University School of Medicine, 1035 Dalgubeol-daero, Dalseo-gu, Daegu 42601, Korea

Tel: $+82-53-258-7747$

Fax: $+82-53-258-4380$

E-mail: hjpark209042@gmail. com
Intracranial hypotension ( $\mathrm{IH})$ is the one of the causes of cerebral venous thrombosis (CVT). ${ }^{1}$ Especially, it has been known that CVT secondary to IH have involve the large cerebral venous sinus with a more acute and severe course. ${ }^{2}$ Currently, anticoagulation has been mainstay for treatment for CVT. However, according to latest study, ${ }^{3}$ vascular recanalization started within the first 8 days of therapeutic anticoagulation. Thus, in case for CVT secondary to $\mathrm{IH}$, which have a more acute and severe course, mechanical thrombectomy may be useful option rather than anticoagulation. Here, we report a case of CVT secondary to IH that was successfully treated using mechanical thrombectomy.

\section{CASE}

A 6o-year-old man presented with an 8 hours history of headache and quadriparesis (Medical Research Council scale scores of $4 / 3$ and $4 / 3$ on the right and left sides, respectively). He had a previous history of lung cancer. A right upper lung lobectomy with resection of the transverse process of $\mathrm{T}_{2}-\mathrm{T}_{5}$ has been performed
15 days prior at another hospital. According to the patient's medical record from the other hospital, he had a history of postural headache that was relieved by hydration and bed rest. He was also diagnosed with tuberculosis and had not taken anti-tuberculosis medication in 8 days. His body temperature was $36.8^{\circ} \mathrm{C}$, blood pressure was $123 / 70 \mathrm{mmHg}$, and pulse rate was $75 / \mathrm{min}$. A cerebrospinal fluid (CSF) study including a cytomorphologic examination to rule out infection and malignany showed normal results.

Magnetic resonance (MR) imaging showed subdural fluid collection with dural thickening on fluid-attenuated inversion recovery imaging (Fig. 1A) and cortical venous engorgement on susceptibility-weighted imaging (Fig. 1B). Based on MR finding, MR venography was done to rule out CVT and demonstrated non-visualization of entire segment of the superior sagittal sinus (SSS) (Fig. 1C). Digital subtraction angiography (DSA) was performed and occlusion of the entire SSS, confluence of sinuses and the left distal transverse sinus with cortical venous reflux was observed (Fig. 1D). As the etiology evaluation of CVT, specific laboratory examination for coagulopathy was done and no evidence for coagulopa- 
thy was noted.

Considering the patient's history of postural headache after resection of the transverse process, radioisotope (RI) cisternography for confirming the any presence of CSF leakage was planned. However, it was not performed because the patient suffered severe headache even with a minimal positional change. Instead of RI cisternography, thoracic spine MR demonstrated diffuse patchy meningeal enhancement with epidural fluid collection form the $\mathrm{T}_{2}-\mathrm{T}_{4}$ spine segment on fat saturation T1-enhanced imaging (Fig. 1E).

Direct non-vitamin K oral anticoagulants (DOAC), dabigatran $150 \mathrm{mg}$ bid was administered. An epidural blood patch failed because of the patient's poor cooperation. On the fifth day of dabigatran use, his mental status deteriorated to semicoma and a generalized tonic-clonic seizure developed. Follow-up MR imaging showed progression of the bilateral subdural fluid collection (Fig. 1F). No hemorrhage or downward herniation was observed.

Mechanical thrombectomy (MT) for the occluded venous segment was planned. A 6F shuttle (Shuttle-SL; Cook, Bloomington, IN, USA) was placed at the right internal jugular vein and aspiration thrombectomy was performed using an Ace 68 catheters (Penumbra; Alameda, CA, USA). Large thrombi were extracted and all occluded segments were recanalized (Fig. $1 \mathrm{G}$ ). The patient's neurologic symptoms, except for the severe postural headache, had fully improved. The epidural blood patch was re-tried at the $\mathrm{T}_{2}-\mathrm{T}_{3}$ level. RI cisternography after blood patch showed no evidence of CSF leakage (Fig. $1 \mathrm{H}$ ). On the 3 months follow-up MR venography, the entire occluded segment was well visualized (Fig. 1I) and no neurological symptom was remained.

\section{DISCUSSION}

$\mathrm{IH}$, a syndrome of negative intracranial pressure and important cause of postural headache in young and middle-age people, is caused by CSF loss. ${ }^{4}$ According to Monroe-Kellie doctrine, the sum of the volumes of CSF, intracranial blood, and brain tissue should be constant in the intact cranium. Thus, if CSF is lost from the spine, intracranial blood volume would increase as a compensatory mechanism. ${ }^{5}$ Thus, some of the characteristics features of IH such as pachymeningeal enhancement, venous engorgement, and subdural fluid collection could developed. ${ }^{4}$ In particular, venous engorgement causes slowing of the venous blood flow. ${ }^{6}$ Reduced flow velocity in the cerebral venous system leads to increased blood viscosity, disrupted blood drainage, and finally CVT and cerebral venous infarction. ${ }^{1}$

CVT had been considered a more lethal and fatal disease than stroke in the arterial system. Despite the medical treatment, death or severe disability occurs in about $15 \%$ of patients and the mortality rate can reach $30 \% .^{3}$ In particular, CVT secondary to IH has a higher tendency to involve the large cerebral venous sinus, such as the SSS. Thus, CVT secondary to IH reportedly involved a more rapid and severe course illness, including higher rates for venous infarction, and National Institutes of Health Stroke Scale score than those of CVT not associated with $\mathrm{IH}^{2}$

In terms of treatment for CVT secondary to IH, treatment consensus is lacking. However, priority was given to CVT treatment in most previously reported cases, because IH was thought to have a benign course but CVT was considered life-threatening. Thus, anticoagulation using heparin or warfarin, recommended as treatment for CVT, has been widely used as primary treatment in cases of CVT secondary to IH. ${ }^{3}$ Nowadays, dabigatran etexilate, DOAC, showed comparable safety and efficacy to those of the warfarin. ${ }^{7}$ Thus, we used dabigatran $150 \mathrm{mg}$ bid for recanalization of CVT. In our case, despite treatment, the patient deteriorated on the fifth day of dabigatran use. According to a previous study, venous recanalization started within the first 8 days of therapeutic anticoagulation in most patients with CVT. ${ }^{3}$ In addition, CVT secondary to IH had a higher tendency to involve the large cerebral venous sinus and exhibited severe symptoms. ${ }^{2}$ Thus, anticoagulation alone may not be useful for acute deteriorating CVT involving the large venous sinus secondary to $\mathrm{IH}$.

In terms of treatment for $\mathrm{IH}$, considering that the occurrence of CVT is related to specific pathophysiologic changes after CSF leakage, and epidural blood patch for IH is also important. ${ }^{5}$ The patients' $\mathrm{IH}$ in our case was induced by resection of the transverse process of the spine. In cases of $\mathrm{IH}$ due to iatrogenic causes such as lumbar puncture or spine surgery, the importance of 

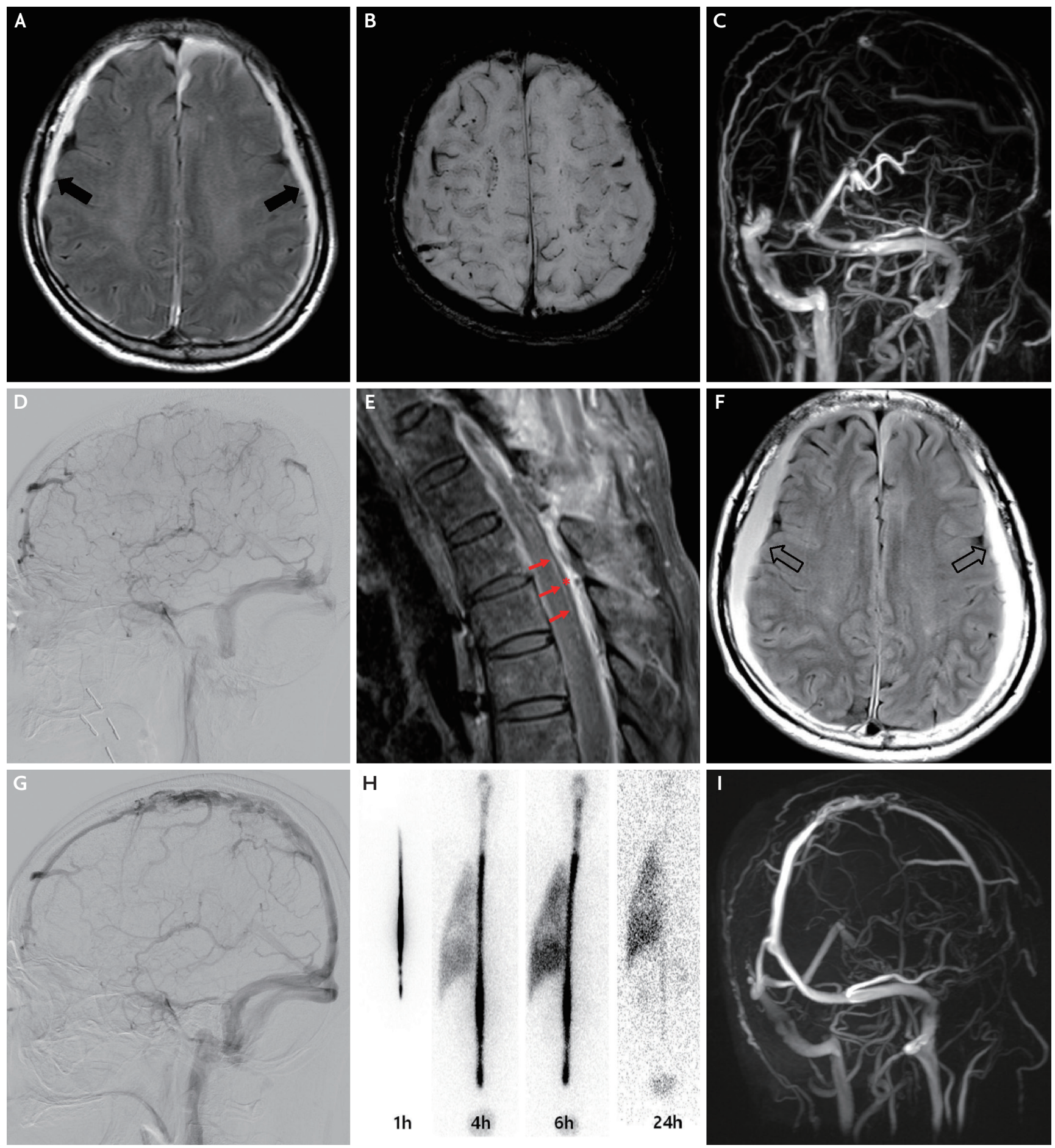

FIG. 1. (A) Subdural fluid collection with dural thickening (black arrows) on bilateral cerebral convexity on magnetic resonance (MR) fluid attenuation inversion recovery (FLAIR) imaging. (B) Cortical venous engorgement on MR susceptibility-weighted imaging. (C) Non-visualization of the entire superior sagittal sinus (SSS) to the left distal transverse sinus (TS) on MR venography. (D) Occlusion of the entire SSS and confluence of the sinuses to the left distal TS with cortical venous reflux on digital subtraction angiography (DSA). (E) Patchy meningeal enhancement (red arrows) and epidural fluid (red asterisk) on fat saturation T1-enhanced imaging. (F) Increased subdural fluid collection (transparent arrows) on bilateral convexity on MR FLAIR imaging after neurological deterioration. (G) Recanalization of the entire occluded cerebral venous sinus after mechanical thrombectomy on DSA. (H) No evidence of cerebrospinal fluid leakage on radioisotope cisternography after epidural blood patch. (I) Good visualization of the entire occluded cerebral venous sinus on MR venography at 3 months after the thrombectomy. 
CSF leakage correction using an epidural blood patch or surgical procedure in refractory case has been emphasized. ${ }^{8}$ However, normalization of pathophysiologic changes takes time. Thus, although the epidural blood patch is essential for CSF leakage correction, its use alone may not be appropriate in case of rapid neurological deterioration.

In our case, the patient's neurological status rapidly deteriorated despite anticoagulation and we did not perform the epidural blood patch because of patient's condition improved dramatically. Nowadays, MT is an alternative and useful option for patients with CVT and neurological worsening despite anticoagulant use. ${ }^{9}$ Considering the clinical features of CVT secondary to IH, MT might be a useful option with anticoagulation and epidural blood patch.

\section{Acknowledgments}

This work was supported by the National Research Foundation of Korea (NRF) grant funded by the Korea government (MSIT) (No. 2020R1G1Aoo7100).

\section{Conflicts of Interest}

No potential conflicts of interest relevant to this article was reported.

\section{REFERENCES}

1. Wilder-Smith E, Kothbauer-Margreiter I, Lämmle B, Sturzenegger M, Ozdoba C, Hause SP. Dural puncture and activated protein $\mathrm{C}$ resistance: risk factors for cerebral venous thrombosis. J Neurol Neurosurg Psychiatry. 1997;63:351356.

2. Honig A, Eliahou R, Pikkel YY, Leker RR. Iatrogenic intracranial hypotension and cerebral venous thrombosis. J Neurol Sci. 2016;366:191-194.

3. Sousa DA, Neto LL, Arauz A, Sousa AL, Gabriel D, Correia $\mathrm{M}$, et al. Early recanalization in patients with cerebral venous thrombosis treated with anticoagulation. Stroke. 2020;51:1174-1181.

4. Schievink WI. Spontaneous spinal cerebrospinal fluid leaks and intracranial hypotension. JAMA. 2006;295:2286-2296.

5. Zhang D, Wang J, Zhang Q, He F, Hu X. Cerebral venous thrombosis in spontaneous intracranial hypotension: a report on 4 cases and a review of the literature. Headache. 2018;58:1244-1255

6. Schievink WI, Maya MM. Cerebral venous thrombosis in intracranial hypotension. Headache. 2008;48:1511-1519.

7. Ferro JM, Coutinho JM, Dentali F, Kobayashi A, Alasheev A, Canhão $\mathrm{P}$, et al. Safety and efficacy of dabigatran etexilate vs dose-adjusted warfarin in patients with cerebral venous thrombosis. JAMA. 2019;76:1457-1465.

8. Perry A, Graffe CS, Brinjikji W, Copeland III WR, Rabinstein AA, Link MJ, et al. Spontaneous occult intracranial hypotension precipitating life-threatening cerebral venous thrombosis: case report. J Neurosurg Spine. 2018;28:669-678.

9. Ilyas A, Chen C-J, Raper DM, Ding D, Buell T, Mastorakos P, Liu KC, et al. Endovascular mechanical thrombectomy for cerebral venous sinus thrombosis: a systematic review. J Neurointerv Surg. 2017;9:1086-1092. 